Badawy, Ahmed and McInnes, Colin (2006) Autonomous structure assembly using potential field functions. In: 57 th International Astronautical Congress, 02-06 Oct 2006, Valencia, Spain.

Strathprints is designed to allow users to access the research output of the University of Strathclyde. Copyright (C) and Moral Rights for the papers on this site are retained by the individual authors and/or other copyright owners. Users may download and/or print one copy of any article(s) in Strathprints to facilitate their private study or for non-commercial research. You may not engage in further distribution of the material or use it for any profitmaking activities or any commercial gain. You may freely distribute the url (http://eprints.collr.strath.ac.uk) of the Strathprints website.

Any correspondence concerning this service should be sent to The Strathprints Administrator: eprints@cis.strath.ac.uk 


\title{
IAC-06- C1.P.3.04
}

\section{Autonomous Structure Assembly Using Potential Field Functions}

\author{
Ahmed Badawy \\ Department of Mechanical Engineering, University of Strathclyde, Glasgow, UK \\ ahmed.badawy@strath.ac.uk \\ Colin R Mclnnes \\ Department of Mechanical Engineering, University of Strathclyde, Glasgow, UK \\ colin.mcinnes@strath.ac.uk
}

\begin{abstract}
The work in this paper aims to introduce a new technique for artificial potential field methods based on a superquadric representation of the obstacle shapes, and a quaternion representation of the object orientation. The superquadric functions facilitate the representation of the actual shape of the obstacles to provide additional free space for the motion of the maneuvering objects, consequently reducing maneuvering effort for these objects. The quaternion representation overcomes the singularities produced when using Euler angles and is more convenient for real time implementation. Potential field methods provide a robust means of generating distributed controls for mobile robots (terrestrial or space) to enable automated assembly tasks. The problem of local minimum formation when dealing with objects having straight edges is also addressed in this paper through changing the superquadric function shape simultaneously in accordance with the relative position and orientation of the objects. The proposed potential function enables maneuvering objects to decide which motion, both in translation and rotation, leads to the quickest descent of the artificial potential most effectively. These coupled translation/rotation manoeuvres show significant benefits over decoupled translation maneuvers.
\end{abstract}

\section{INTRODUCTION}

Autonomous assembly is a key behavior which is applied on almost all scales and is present in a range of biological and physical processes. The objective of the autonomous assembly process is to allow a group of elements to behave in a coordinated way by following an algorithm. The techniques of autonomous assembly could be used in large space structure assembly where it is dangerous and costly to use human effort.

The potential field technique used herein aims to provide a motion planning algorithm to reach a goal configuration without collision between the moving elements of the structure 1 . The notion of this method is a physical idea for which any system tends to a stable state, such as cooling a liquid to a periodic crystal structure.

The work presented in this paper uses the potential field method in structure assembly through a superquadric representation of each element to simulate its actual shape and offer the maximum free work space for the motion of a large number of elements. The deficiency of the superquadric obstacle representation in determining the separation distance for specific configurations and interpolating for others was overcome through using quaternions for orientation representation. 


\section{QUATERNIONS AND SUPERQUADRICS}

Consider, for simplicity, two parallelepiped elements in motion from some initial configuration toward a goal. Each is considered as an obstacle to the other. Knowing the Cartesian coordinate as well as the quaternion parameters for both of them, it is possible to calculate the relative position of one with respect to the other using quaternion algebra through the homogeneous transformation matrix $\tau$, by considering that the origin of the rotating frame is fixed at the centre of the obstacle ${ }^{2}$.

$$
\begin{aligned}
& \boldsymbol{\tau}= \\
& {\left[\begin{array}{ccc}
q_{1}^{2}-q_{2}^{2}-q_{3}^{2}+q_{4}^{2} & 2\left(q_{1} q_{2}+q_{3} q_{4}\right) & 2\left(q_{1} q_{3}-q_{2} q_{4}\right) \\
2\left(q_{1} q_{2}-q_{3} q_{4}\right) & -q_{1}^{2}+q_{2}^{2}-q_{3}^{2}+q_{4}^{2} & 2\left(q_{2} q_{3}+q_{1} q_{4}\right) \\
2\left(q_{1} q_{3}+q_{2} q_{4}\right) & 2\left(q_{2} q_{3}-q_{1} q_{4}\right) & -q_{1}^{2}-q_{2}^{2}+q_{3}^{2}+q_{4}^{2}
\end{array}\right]}
\end{aligned}
$$

where the quaternion parameters in this transformation are the current quaternion parameters of the obstacle. Hence, the relative coordinates $\mathrm{x}, \mathrm{y}$, and $\mathrm{z}$ can be expressed and used in the implicit equation of the superquadric surface ${ }^{3,4}$

$$
\left[\left(\frac{x}{a}\right)^{\frac{2}{\varepsilon_{2}}}+\left(\frac{y}{b}\right)^{\frac{2}{\varepsilon_{2}}}\right]^{\frac{\varepsilon_{2}}{\varepsilon_{1}}}+\left(\frac{z}{c}\right)^{\frac{2}{\varepsilon_{1}}}=1
$$

where $a, b$, and $c$ define the size of the obstacle in the $\mathrm{x}, \mathrm{y}$, and $\mathrm{z}$ direction respectively, $\varepsilon_{1}$ defines the obstacle shape in the z-direction and $\varepsilon_{2}$ defines its shapes in the $x-y$ plane.

Through changing the five parameters, $a, b$, $c, \varepsilon_{1}$ and $\varepsilon_{2}$, the superquadric representation of the obstacle can be changed from the actual obstacle shape near the obstacle edges to a sphere far from its edges. This is the parametric property of superquadrics.

Before defining the obstacle potential energy, it is necessary to define the distance between any point and the superquadric surface.

The "inside-outside" function which defines if the point lies inside, on the surface or outside the superquadric shape is given by ${ }^{5}$ :

$$
F(\mathbf{a}, x, y, z)=\left[\left(\frac{x}{a}\right)^{\frac{2}{\varepsilon_{2}}}+\left(\frac{y}{b}\right)^{\frac{2}{\varepsilon_{2}}}\right]^{\frac{\varepsilon_{2}}{\varepsilon_{1}}}+\left(\frac{z}{c}\right)^{\frac{2}{\varepsilon_{1}}}
$$

where a represents the five size and shape parameters. If the value of $F(\mathbf{a}, x, y, z)=1$, the point $(x, y, z)$ lies on the surface of the superquadric. If $F(\mathbf{a}, x, y, z)>1$, the point lies outside and if $F(\mathbf{a}, x, y, z)<1$ the point lies inside the superquadric

The main need to calculate the obstacle potential energy is to define the distance between any point in space and the superquadric surface taking into consideration that the origin of the Cartesian coordinate system will be the centre of the obstacle itself.

The minimum distance calculation through the Euclidian distance method is computationally too expensive ${ }^{6}$. The most efficient approximation for the distance between a point and the superquadric shape is based on the implicit form of the superquadric and is given as ${ }^{7,8}$ :

$$
d(\mathbf{a}, x, y, z)=\mid \mathbf{r}\left(1-F(\mathbf{a}, x, y, z)^{\frac{-\varepsilon_{1}}{2}}\right)
$$

where a represent the superquadric size parameters and $x, y$ and $z$ are the point coordinates with respect to the frame of reference centered on the centre of the superquadric shape. The parameters $\varepsilon_{1}$ and $\varepsilon_{2}$ should be chosen to vary from their values that represent the obstacle shape to that for the spherical shape. The way in which these parameters change depends only on the obstacle shape. Points at an equal distance from the obstacle will show equal obstacle potential. Figure 1 shows how iso-potential contours change their shapes from the obstacle shape to a spherical one as the distance increases from the obstacle edges.

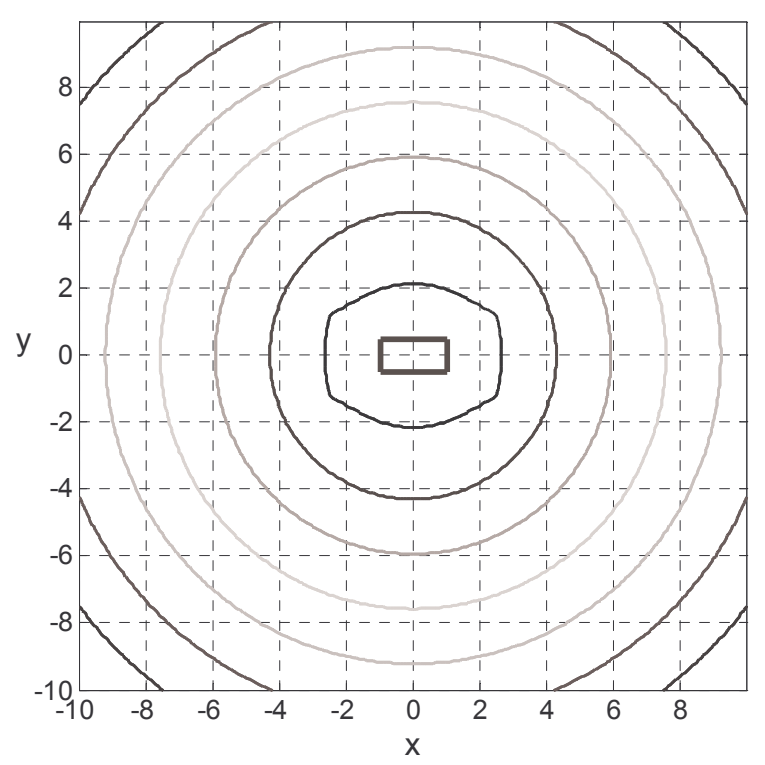

Fig. 1: Obstacle iso-potential contours 


\section{ATTRACTIVE POTENTIAL FUNCTION}

Consider a maneuvering rigid body which performs pure rotation in the body frame of reference, is aligned with the principal axes of inertia of the rigid body and rotates with angular velocity $\omega$ with respect to an inertial frame of reference. The product moments of inertia are equal to zero and the mass moment of inertia of the rigid body is constant. The inertia matrix is then:

$$
\mathbf{I}=\left[\begin{array}{ccc}
I_{1} & 0 & 0 \\
0 & I_{2} & 0 \\
0 & 0 & I_{3}
\end{array}\right]
$$

The angular momentum is then defined as:

$$
\mathbf{H}=\mathbf{I} \boldsymbol{\omega}
$$

$$
\text { where } \boldsymbol{\omega}=\left\lceil\omega_{1} \omega_{2} \omega_{3}\right\rceil^{T} \text {. }
$$

From Euler's equation the external torque acting on the rigid body is:

$$
\begin{aligned}
\mathbf{T} & =\dot{\mathbf{H}} \\
& =\dot{\mathbf{H}}_{b}+\boldsymbol{\omega} \times \mathbf{H} \\
\mathbf{T} & =\mathbf{I} \dot{\boldsymbol{\omega}}+\boldsymbol{\omega} \times \mathbf{I} \boldsymbol{\omega}
\end{aligned}
$$

The control torque $\mathbf{T}$ and translation velocity $\mathbf{v}$ to maneuver such a rigid body for assembly will now be considered using an artificial potential function. The potential function based on Lyapunov's second method will be defined as:

$\mathrm{V}=\frac{1}{2}\left|\mathbf{r}-\mathbf{r}_{G}\right|^{2}+C_{1}\left(\overline{\mathbf{q}}^{T} \overline{\mathbf{q}}\right)+\frac{1}{2} \boldsymbol{\omega}^{T} \mathbf{I} \boldsymbol{\omega}$

where $C_{1}$ is the control torque gain and $\overline{\mathbf{q}}$ is the vector of the error quaternions $\left\lceil q_{1} q_{2} q_{3}\right\rceil^{T}$. The fourth quaternion parameter, $q_{4}=\sqrt{1-q_{1}^{2}-q_{2}^{2}-q_{3}^{2}}$, will reach its goal value, $q_{4}=1$, as the first three terms reach zero.

It is clear that the proposed function satisfies the condition of a Lyapunov function such that $\mathrm{V}=0$ at the goal position where $\boldsymbol{\omega}$ and $\overline{\mathbf{q}}$ are both zero and $\mathrm{V}>0$ for every state vector except at the goal position.

The time derivative of the function $\mathrm{V}$ is:

$$
\dot{\mathrm{V}}=\left|\mathbf{r}-\mathbf{r}_{G}\right| \dot{\mathbf{r}}+2 C_{1}\left(\dot{\overline{\mathbf{q}}}^{\mathbf{T}} \overline{\mathbf{q}}\right)+\boldsymbol{\omega}^{\mathbf{T}} \mathbf{I} \dot{\boldsymbol{\omega}}
$$

The first derivative of the quaternion is defined as ${ }^{9}$ :

$$
\dot{\overline{\mathbf{q}}}=\frac{1}{2} \mathbf{Q} \mathbf{\omega}
$$

where $\mathbf{Q}$ is the matrix of quaternion components and is defined as:

$\mathbf{Q}=\left[\begin{array}{ccc}q_{4} & q_{3} & -q_{2} \\ -q_{3} & q_{4} & q_{1} \\ q_{2} & -q_{1} & q_{4}\end{array}\right]$

Substituting in (9)

$$
\begin{aligned}
\dot{\mathbf{V}} & =\left|\mathbf{r}-\mathbf{r}_{G}\right| \dot{\mathbf{r}}+C_{1} \boldsymbol{\omega}^{\mathbf{T}} \mathbf{Q}^{\mathbf{T}} \overline{\mathbf{q}}+\boldsymbol{\omega}^{\mathbf{T}} \mathbf{I} \dot{\boldsymbol{\omega}} \\
& =\left|\mathbf{r}-\mathbf{r}_{G}\right| \dot{\mathbf{r}}+\boldsymbol{\omega}^{\mathbf{T}}\left(C_{1} \mathbf{Q}^{\mathbf{T}} \overline{\mathbf{q}}+\mathbf{I} \dot{\boldsymbol{\omega}}\right)
\end{aligned}
$$

and so we have $\mathbf{Q}^{\mathbf{T}} \overline{\mathbf{q}}=q_{4} \overline{\mathbf{q}}$ and then:

$$
\dot{\mathrm{V}}=\left|\mathbf{r}-\mathbf{r}_{G}\right| \dot{\mathbf{r}}+\boldsymbol{\omega}^{\mathbf{T}}\left(C_{1} q_{4} \overline{\mathbf{q}}+\mathbf{I} \dot{\boldsymbol{\omega}}\right)
$$

From (7)

$$
\dot{\mathrm{V}}=\left|\mathbf{r}-\mathbf{r}_{G}\right| \dot{\mathbf{r}}+\boldsymbol{\omega}^{\mathbf{T}}\left(C_{1} q_{4} \overline{\mathbf{q}}+\mathbf{T}-\boldsymbol{\omega} \times \mathbf{I} \boldsymbol{\omega}\right)
$$

Let $\mathbf{T}$, the linear control torque be $\mathbf{T}=-C_{1} q_{4} \overline{\mathbf{q}}-C_{2} \boldsymbol{\omega}$ so that:

$$
\dot{\mathrm{V}}=\left|\mathbf{r}-\mathbf{r}_{G}\right| \dot{\mathbf{r}}+\boldsymbol{\omega}^{\mathbf{T}}\left(-C_{2} \boldsymbol{\omega}-\boldsymbol{\omega} \times \mathbf{I} \boldsymbol{\omega}\right)
$$

But $\omega^{\mathbf{T}}(\boldsymbol{\omega} \times \mathbf{I} \boldsymbol{\omega})=0$ so that

$$
\dot{\mathrm{V}}=\left|\mathbf{r}-\mathbf{r}_{G}\right| \dot{\mathbf{r}}-C_{2} \boldsymbol{\omega}^{\mathbf{T}} \boldsymbol{\omega}
$$

Then let

$$
\mathbf{v}=-k \nabla \mathrm{V}|\nabla \mathrm{V}|^{-1}
$$

where $\mathbf{v}$ is the velocity of the maneuvering object, and $k$ expressed in equation (16) is chosen as $k=v_{\max }\left(1-e^{-\beta \mathrm{V}}\right)$, where $v_{\max }$ is the maximum controlled velocity, and $\beta$ is a constant $^{10,11}$.

Hence the last condition, $\dot{\mathrm{V}}<0$, is satisfied for all state vectors except at the goal position, so the proposed function can be considered as a Lyapunov function providing the following relation is valid:

$$
\dot{\boldsymbol{\omega}}=-\mathbf{I}^{-1}\left(C_{1} q_{4} \overline{\mathbf{q}}+C_{2} \boldsymbol{\omega}+\boldsymbol{\omega} \times \mathbf{I} \boldsymbol{\omega}\right)
$$




\section{REPULSIVE POTENTIAL FUNCTION}

Local minima can appear in some obstacle representations due to the interaction between the iso-potential contours of both the goal and obstacle, where one is spherical while the other has straight edges. The superquadric potential proposed by Volpe ${ }^{12,13}$, overcomes this problem of local minimum with a single obstacle. The deformable superquadric function used by Volpe changes its shape from the actual obstacle shape near its edges to a spherical shape far from the obstacle. The superquadric implicit function for a parallelepiped can be written as:

$F(\mathbf{a}, x, y, z)=\left(\frac{x}{a}\right)^{2 m}+\left(\frac{y}{b}\right)^{2 m}+\left(\frac{z}{c}\right)^{2 m}$

where, $m$ varies from infinity at the obstacle edges to unity sufficiently far from them.

The formulation of the obstacle repulsive potential energy function depends on the required controlled object behavior whilst approaching the obstacle. Two types of repulsive potential function are used herein: the avoidance potential and the approach potential.

The idea of the avoidance potential is to prevent collision between the controlled object and the surrounding obstacles by introducing infinite repulsive energy around the obstacle to force the controlled object to move away from the obstacle regardless of the kinetic energy of each of them.

Through measurement of the minimum distance between the two objects, it is possible to use the Born approximation for a Yukawa potential ${ }^{14}$ in which the exponential term reaches zero faster than the $1 / d$ term.

$V_{o b s_{i, j}}=A_{j} \frac{e^{-\alpha d_{i, j}}}{d_{i, j}}, \quad d_{i, j} \geq 1$

The distance between object $j$ and obstacle $i$, $d_{i, j}$, is obtained from the superquadric separation distance using equation (4). The parameter $\alpha$ controls the sharpness of the obstacle potential shape and the transition of the isopotential contour shape changing from the actual obstacle shape to the spherical one. Increasing the value of $\alpha$ increases the sharpness of the potential decay, this limits the distance of influence of the obstacle on the overall potential. The choice of the value of $\alpha$ is largely dependant on the number of objects, besides changing its value can help in overcoming the formation of local minima, produced when dealing with many objects. The parameter $\alpha$ will therefore be expressed as a function of the minimum distance between the objects as well as the object priority when moving.

The definition of the parameter $A$ is crucial in structure assembly problems. It will be expressed as a function of the configuration of the objects, allowing the obstacle potential to decay to zero at the goal configuration to allow for smooth contact which is required for connection of the structure elements ${ }^{9}$.

The approach potential objective is to decrease the kinetic energy of the moving object when approaching obstacles with a certain limit to reduce the contact velocity. The approach potential function can be expressed as:

$$
V_{o b s i, j}=A_{j} e^{-\alpha d_{i, j}{ }^{1+\frac{1}{\alpha}}}, d<1
$$

\section{OVERALL POTENTIAL FUNCTION}

The total potential is a linear superposition of the attractive and repulsive potentials. The total potential of maneuvering object $j$, in the presence of $n$ obstacles is given as:

For $d \geq 1$

$$
\begin{aligned}
\mathrm{V}_{j} & =\frac{1}{2}\left|\mathbf{r}_{j}-\mathbf{r}_{G, j}\right|^{2}+C_{1}\left(\overline{\mathbf{q}}_{j}{ }^{T} \overline{\mathbf{q}}_{j}\right)+\frac{1}{2} \boldsymbol{\omega}_{j}{ }^{T} \mathbf{I}_{j} \boldsymbol{\omega}_{j} \\
& +\sum_{i=1, i \neq j}^{n} A_{j}\left(\mathbf{r}_{j}\right) \frac{e^{-\alpha\left(\mathbf{r}_{j}, \mathbf{r}_{i}\right) d_{j, i}\left(\mathbf{a}, \mathbf{r}_{j}, \mathbf{r}_{i}\right)}}{d_{j, i}\left(\mathbf{a}, \mathbf{r}_{j}, \mathbf{r}_{i}\right)}
\end{aligned}
$$

$d<1$

$$
\begin{aligned}
\mathrm{V}_{j} & =\frac{1}{2}\left|\mathbf{r}_{j}-\mathbf{r}_{G, j}\right|^{2}+C_{1}\left(\overline{\mathbf{q}}_{j}{ }^{T} \overline{\mathbf{q}}_{j}\right)+\frac{1}{2} \boldsymbol{\omega}_{j}{ }^{T} \mathbf{I}_{j} \boldsymbol{\omega}_{j} \\
& +\sum_{i=1, i \neq j}^{n} A_{j}\left(\mathbf{r}_{j}\right) e^{-\alpha\left(\mathbf{r}_{j}, \mathbf{r}_{i}\right) d_{j, i}\left(\mathbf{a}, \mathbf{r}_{j}, \mathbf{r}_{i}\right)^{1+\frac{1}{\alpha\left(\mathbf{r}_{j}, \mathbf{r}_{i}\right)}}}
\end{aligned}
$$

The element velocity vector is now required to be in the negative gradient in the direction of the potential field. Substituting in equation (16) for the desired velocity we obtain:

$$
\mathbf{v}_{j}=\frac{-v_{\max }\left(1-e^{-\beta \mathrm{V}_{a t t, j}}\right)}{\left|\nabla \mathrm{V}_{j}\right|}\left|\mathbf{r}_{j}-\mathbf{r}_{G_{j}}\right| \nabla \mathbf{r}_{j}+\sum_{i=1, i \neq j}^{m} \frac{\partial \mathrm{V}_{o b s_{i, j}}}{\partial d_{i, j}} \nabla d_{i, j}
$$


where $v_{\max }$ is the maximum controlled velocity, which is a function of the characteristics of maneuvering object.

The radial Euclidian distance, $d$, as expressed before depends on the relative distances between the obstacle and the controlled object in an obstacle body frame of reference. This distance is calculated, again, from the homogeneous transformation using quaternions as:

$$
\left[\begin{array}{l}
x \\
y \\
z
\end{array}\right]=\boldsymbol{\tau}\left[\begin{array}{c}
x_{o b j}-x_{o b s} \\
y_{o b j}-y_{o b s} \\
z_{o b j}-z_{o b s}
\end{array}\right]
$$

Substituting the values of $x, y$, and $z$ in the superquadric inside-outside function and the radial Euclidian distance equation we can obtain the term $\nabla d$, which provides the link between the translational and rotational motion.

\section{NUMERICAL RESULTS}

The numerical results presented herein are the assembly of a cube using four parallelepipeds, which may be considered as a repeated unit in large space frame structure assembly. The elements are assumed to be made from steel with $1 \mathrm{~m}$ length, and $0.2 \times 0.2 \mathrm{~m}$ cross section. The control gain was chosen as unity.

The starting position is shown in fig(2-a), and subsequent motion is demonstrated in fig $(2-b, 2-c)$. The final configuration is shown in fig(2-d).

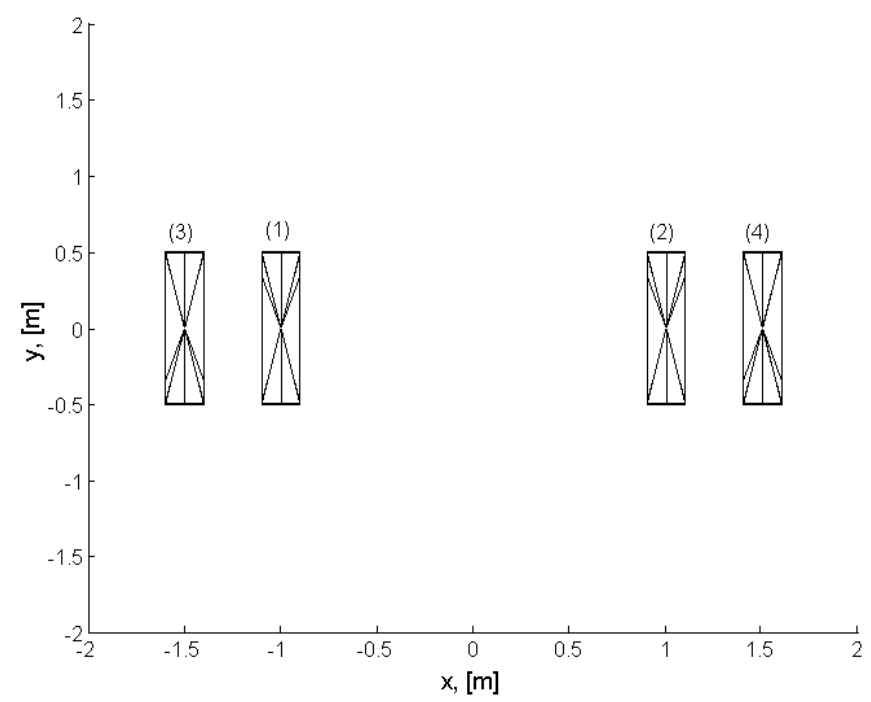

Fig. 2.a: The start position, $\mathrm{t}=0 \mathrm{sec}$

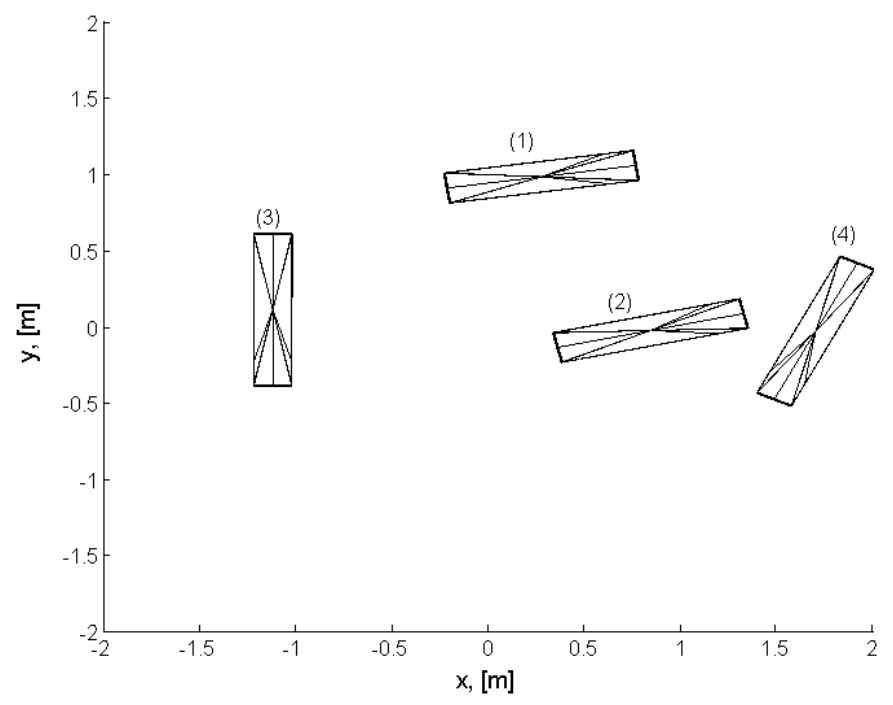

Fig. 2.b: $\mathrm{t}=2.4 \mathrm{sec}$

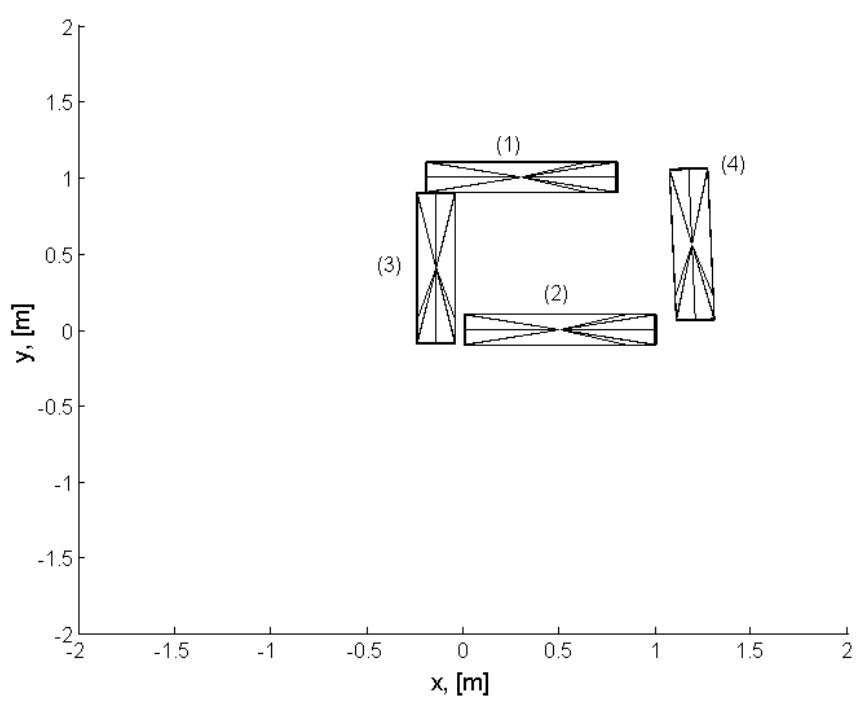

Fig. 2.c: $t=25 \mathrm{sec}$

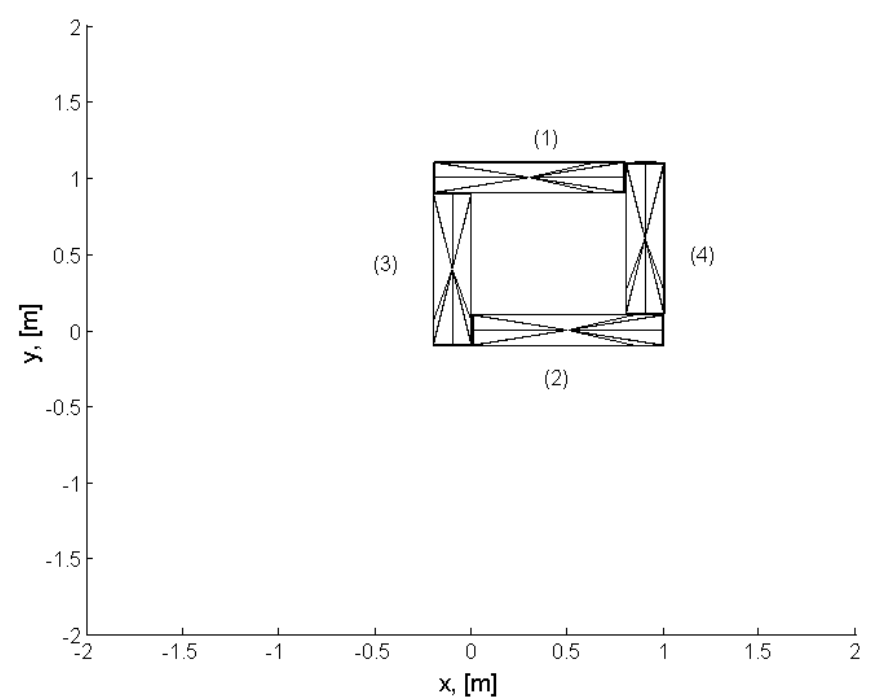

Fig. 2.d: The assembled structure, $t=200 \mathrm{sec}$ Fig. 2: Assembling of a 2-D cube 
The mutual effect between position and orientation of the elements is shown in fig(3).

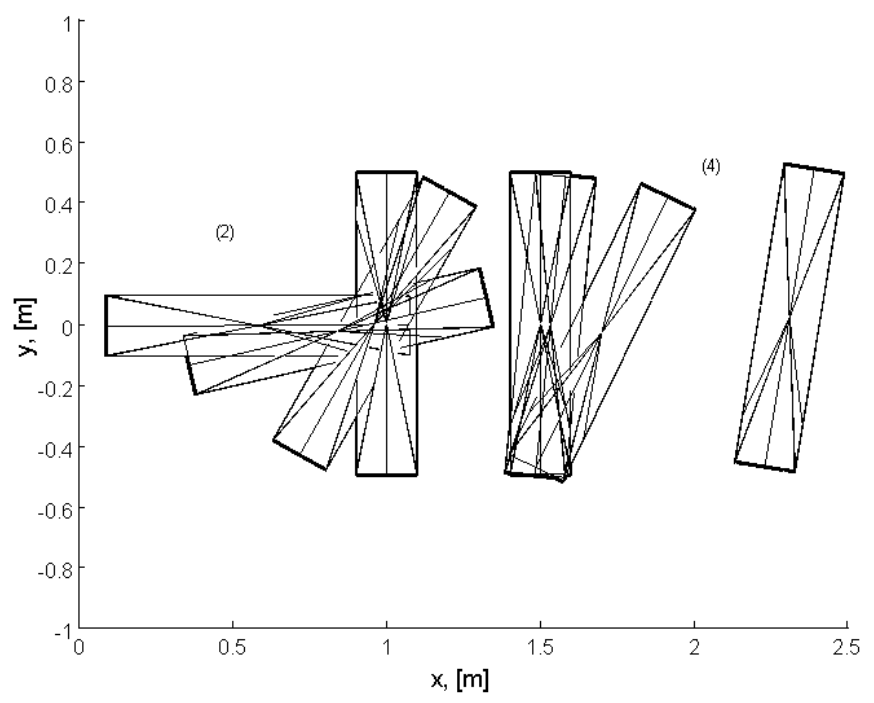

Fig. 3: Proximity motion of two objects (2) and (4)

The control torque, angular velocity, and quaternions of element number (2) are shown in fig. (4).

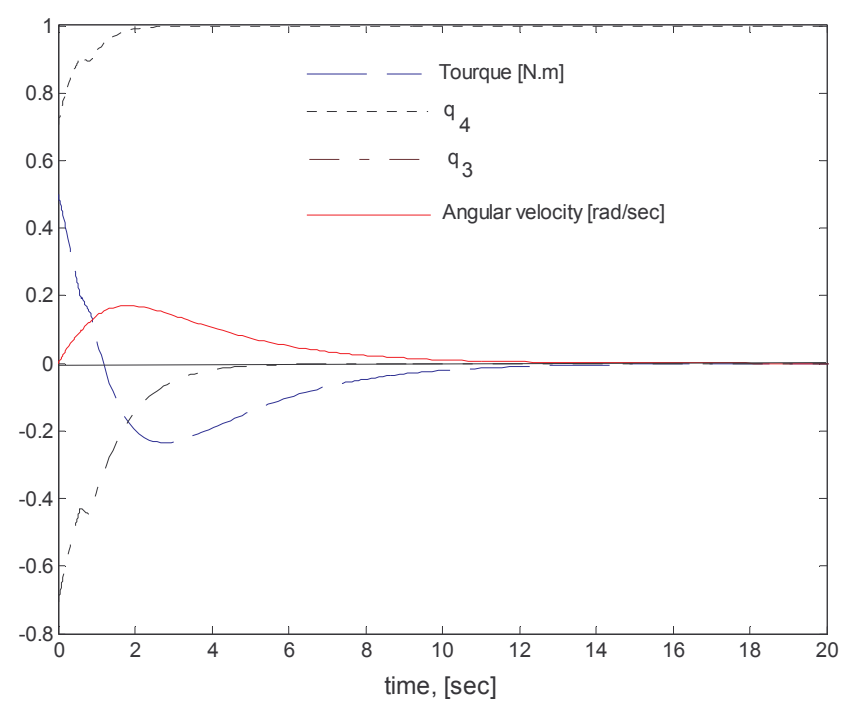

Fig. 4: Control parameters through motion of element number (2)

\section{CONCLUSION}

The utilization of quaternion and superquadrics gives good results in the control of maneuvering objects which are relatively close to each other. Although four simultaneously moving obstacles were considered, no local minimum or goal position shifts occurred due to the simultaneous change in the shape of the superquadric obstacle potential with respect to the separation between any two obstacles.
The bounded time approach gives good results in structure assembly in which the velocity of the maneuvering objects vanishes (and so contact is made) in a finite time.

An attitude change commanded by the required control torque demonstrates that the maneuvering object is capable of choosing between both orientation and translation to decrease the overall potential.

The proposed potential function enables online controllers to choose the path and attitude of maximum negative gradient of the artificial potential. Such on-line controllers could have direct application to the automated assembly of future large space structures.

\section{REFERENCES}

1. Khatib O., "Real-Time Obstacle Avoidance for Manipulators and Mobile Robots", the International Journal of Robotics Research, 1986.

2. Paul R. P., "Robot Manipulators: Mathematics, Programming, and Control" 1981.

3. Barr A. H., "Superquadrics and Anglepreserving Transformations", IEEE Computer Graphics and Applications, vol 1, pp. 11-23, 1981.

4. Bajcsy R., and Solina F., "Three Dimensional Object Representation Revisited", First Int. Computer Vision Conf., London, England, pp. 231-241, 1987.

5. Solina F., and Bajcsy R., "Recovery of Parametric Models from Range Images: The Case for Superquadrics with Global Deformations", IEEE transaction on pattern analysis and machine intelligence, vol. 12, no. 2, pp. 131-147, February 1990.

6. Harden T., "The Implementation of Artificial Potential Field Based Obstacle Avoidance for a Redundant Manipulator", M. Sc thesis, The University of Texas at Austin, 1997.

7. Chevalier L., Jaillet F., and Baskurt A., "Segmentation and Superquadric Modeling of 3D Objects", Journal of WSCG, vol. 11, no. 1, 2003.

8. Badawy A. and Mclnns C.R., " Separation Distance for Robot Motion Control using Superquadric Obstacle Potentials", International Control Conference, Glasgow, Scotland, 2006 
9. Wie B., "Space Vehicle Dynamics and Control", AIAA Education series, 1998.

10. McQuade F., and Mclnnes C.R., "Distributed Control for Serial Assembly in Space", $\quad$ Proc. $\quad 5^{\text {th }} \quad$ International Conference of space'96, vol. 2, pp. 11691175, 1996.

11. McQuade F., "Autonomous Control for On-Orbit Assembly Using Artificial Potential Functions", Ph. D thesis, University of Glasgow, 1997.

12. Volpe R., "Real and Artificial Forces in the Control of Manipulators: Theory and Experiments" Ph. D thesis, 1990.

13. Volpe R., Khosla P.," Manipulator Control with Superquadric Artificial Potential Functions: Theory and Experiments", IEEE Transaction on Systems, Man, and Cybernetics, 1990.

14. Cohen C., T., Diu B., and Laloë F., "Quantum Mechanics", volume 2. John Wiley and Sons, New York, 1977. 